\title{
The metallicity-luminosity relationship of dwarf irregular galaxies
}

\section{A new approach}

\author{
A. M. Hidalgo-Gámez ${ }^{1, \star}$, F. J. Sánchez-Salcedo ${ }^{2}$, and K. Olofsson ${ }^{1}$ \\ 1 Astronomiska observatoriet, Box 515, 75120 Uppsala, Sweden \\ e-mail: anamaria@astro.uu.se, kjell.olofsson@astro.uu.se \\ 2 Instituto de Astronomía-UNAM, Ciudad Universitaria, Apt. Postal 70 264, C.P. 04510, Mexico City, Mexico \\ e-mail: jsanchez@astroscu.unam.mx
}

Received 21 June 2001 / Accepted 21 November 2002

\begin{abstract}
The nature of a possible correlation between metallicity and luminosity for dwarf irregular galaxies, including those with the highest luminosities, has been explored using simple chemical evolutionary models. Our models depend on a set of free parameters in order to include infall and outflows of gas and covering a broad variety of physical situations. Given a fixed set of parameters, a non-linear correlation between the oxygen abundance and the luminosity may be established. This would be the case if an effective self-regulating mechanism between the accretion of mass and the wind energized by the star formation could lead to the same parameters for all the dwarf irregular galaxies. In the case that these parameters were distributed in a random manner from galaxy to galaxy, a significant scatter in the metallicity-luminosity diagram is expected. Comparing with observations, we show that only variations of the stellar mass-to-light ratio are sufficient to explain the observed scattering and, therefore, the action of a mechanism of self-regulation cannot be ruled out. The possible origin of discrepancies in the metallicity-luminosity correlation found by different authors is discussed.
\end{abstract}

Key words. ISM: abundances - galaxies: dwarf - galaxies: ISM - galaxies: irregular

\section{Introduction}

A strong correlation between the metallicity and the luminosity, two of the global basic parameters of a galaxy, could be helpful in order to understand, e.g., the evolution of galaxies. Additionally, a tight correlation between these parameters may be useful in the determination of the mass content of these objects (Gallagher \& Wyse 1994), provided the massluminosity $(M / L)$ ratio is constant. Also, as the metallicity may be lower for galaxies of low luminosity, the existence of a correlation could be very useful in the search for very low metallicity galaxies (Salzer 1998) which could contribute to our understanding of galaxy formation. Finally, such a correlation could be used, at least to a first approximation, to derive the metallicity of a dwarf irregular galaxy from its absolute magnitude (distance) and vice versa.

Several contradictory results regarding the existence of a correlation between the metallicity and the luminosity (hereafter the $Z-L$ relationship) of dwarf galaxies have been presented over the years. Aaronson \& Mould (1985) found a strong correlation for dwarf spheroidal galaxies. On the other hand, the weak correlations found for blue compact

Send offprint requests to: A. M. Hidalgo-Gámez,

e-mail: anamaria@astroscu.unam.mx

* Present address: Instituto Politécnico Nacional, Mexico City, Mexico; e-mail: ana@esfm.ipn.mx galaxies (BCGs) (Campos-Aguilar et al. 1993; Peña \& Ayala 1993) and low surface brightness galaxies (LSBGs) (McGaugh 1994) do not support such a relationship for gas-rich galaxies. Recently, a tight correlation was established for a sample of low surface brightness dwarf irregular galaxies (van Zee et al. 1997). The relationship between metallicity and luminosity of dwarf irregular galaxies (hereafter dI) was first suggested by Kinman \& Davidson (1981), based on the metallicity-mass relationship studied by Lequeux et al. (1979). Subsequently, this parameter space was investigated, among others, by Skillman et al. (1989) (hereafter SKH). Recently, Richer \& McCall (1995, hereafter RM) and Hidalgo-Gámez \& Olofsson (1998, hereafter Paper I) presented a weaker $Z-L$ relationship for dIs, which is more similar to those obtained for other classes of gas-rich dwarf galaxies. To summarize, the existence of an empirical $Z-L$ relationship for dwarf irregular galaxies is far from unambiguous, but a weak trend cannot be ruled out completely.

An anticorrelation between the metallicity and the fraction of the mass of gas is expected in the closed-box scenario (e.g. Tinsley 1978; van Zee et al. 1997; Pilyugin \& Ferrini 2000a,b). However, the infall and outflow of material may weaken or even change significantly this dependence. Galactic winds may exert an important effect on this correlation. For small galaxies with relatively small potential field, supernova explosions may be energetic enough to eject the heavy elements thus reinforcing the mentioned anticorrelation between 
the gas fraction and the metallicity. In recent years, many investigations on the ejection of heavy elements have been carried out with contradictory results (e.g. MacLow \& Ferrara 1999; Strickland \& Stevens 2000; Silich \& Tenorio-Tagle 2001). For instance, Recchi et al. (2001) concluded that when a dark matter component is included, the metals never leave the parent galaxy, but follow a champagne-flow trajectory. The observational $Z-L$ relationship could help to constrain the rate of infall and outflows, but the situation is still confusing. While Matteucci \& Chiosi (1983) make use of those processes to explain the large scatter of $Z$ against the mass fraction of gas, Pilyugin \& Ferrini (2000b) argue that a linear $Z-L$ relationship can be expected from irregular to spirals, probably even if infall or ejection of gas were included.

In order to study this problem, a detailed investigation combining chemical modeling and observational data is performed in this paper. In the next section we describe different evolutionary models and the theoretical basis to invoke a $Z-L$ relationship. In Sect. 3 the observational data on a subsample of dIs are presented. In Sect. 4 a comparison between the theoretical $Z-L$ relationship and observations is carried out. The consistency of the models and other implications are analyzed, for a particular case, in Sect. 5. A discussion on the origin of the differences between the $Z-L$ found by different authors is presented in Sect. 6. A summary of the conclusions is given in Sect. 7.

\section{The theoretical $Z-L$ relationship}

The various $Z-L$ relationships derived so far have been based on observational data, being the best known of such correlations those obtained for irregular galaxies. In this investigation, the study will be restricted to the so-called dwarf irregulars. According to Paper I, dwarf irregular galaxies are those with absolute magnitude in the $B$-band smaller than -17 and an optical radius smaller than $3 \mathrm{kpc}$. Moreover, their surface brightness are located between 20 and $24 \mathrm{mag} / \mathrm{arsec}^{2}$. This last condition was imposed in order to avoid all the blue compact and low surface brightness galaxies. These galaxies might be in a different stage of evolution which may introduce an additional source of scatter in the $Z-L$ diagram.

Pilyugin \& Ferrini (2000a) studied the relationship between the gas fraction and the metallicity based on the simplest of the chemical evolutionary models, the closed-box model, for dwarf irregular galaxies. They concluded that the closed-box approximation is not appropriate to explain the observed relationship (see also Matteucci \& Chiosi 1983). Moreover, the metallicities derived from the closed-box model are always larger than those derived from observations (confirmed in this study).

The effective yield, defined as the yield that would be deduced if the galaxy were assumed to behave as in the simple closed box model, has typical values of $p=0.004$ (e.g. Lequeux et al. 1979). Larger values are found for the galactic $\mathrm{H}_{\text {II }}$ regions and the solar neighbourhood $(p=0.01)$, in agreement with the trend of larger effective yields for more enriched regions (Peimbert \& Serrano 1982). Matteucci \& Chiosi (1983) varied the effective yields by a factor of 10 in order to explain the differences in metallicity between the LMC and I Zw 18. On the other hand, Vila-Costas \& Edmunds (1992) found that a variation by a factor of 3 in the effective yields would explain the metallicity gradients in spiral galaxies. These variations in the yield might indicate the weakness of the closed-box model for explaining the evolution of gas-rich galaxies.

\subsection{Description of the chemical evolutionary models and the relationship between metallicity and gas mass fraction}

In this section, it will be shown that the relationship between the metal content of a galaxy and its mass fraction of gas is not necessarily linear. The main goal of the present investigation is to explore the physical requirements that would lead to a relationship between the luminosity and the metallicity and the possible sources of scattering. For this purpose we consider a rather realistic situation in which the infall and outflow of gas are allowed, in contrast with the traditional closed-box model.

The starting point will be the following general equations on the chemical evolution of a galaxy (see, for example, Ferrara \& Tolstoy 2000)

$\frac{\mathrm{d} m_{\mathrm{g}}}{\mathrm{d} t}=-\psi(t)+E+A(t)-W(t)$

and

$\frac{\mathrm{d}}{\mathrm{d} t}\left[m_{\mathrm{g}} Z\right]=-Z[\psi(t)+W(t)]+E_{i}+Z^{\prime} A(t)$

where $m_{\mathrm{g}}$ is the total mass of gas, $Z$ stands for the metallicity of the galaxy, $A(t)$ is the rate of infall gas with a certain metallicity $Z^{\prime}, W(t)$ is the rate of outflows with the same metallicity as the parent galaxy, $\psi$ is the star formation rate, and in the instantaneous recycling approximation (IRA) $E=R \psi$ and $E_{i}=\left[R Z+y_{z}(1-R)\right] \psi$, with $R$ the fraction of the mass transformed into stars which is returned to the interstellar medium and $y_{z}$ the yield of the metals. Note that $\psi(t), A(t)$ and $W(t)$ are either positive or zero. The assumption that inflow and outflow occur simultaneously is often adopted in semi-analytical models of galaxy formation (e.g. Cole et al. 1994; Somerville \& Primack 1999; Efstathiou 2000) and it is well justified if the infalling gas is clumpy.

The limitations of the IRA are discussed in Tinsley (1980). The application of this approximation may be controversial because for some chemical elements, like carbon, is not very realistic (Skillman 1999). Some authors have solved the equations above without assuming IRA (Matteucci \& Chiosi 1983; Chiosi 1986), whereas some others have applied it only to oxygen (Peimbert \& Serrano 1982; Skillman 1999). Since the present investigation deals only with oxygen (see Sect. 3), the IRA is justified. However, the results should be interpreted with caution if the model should be applied to some other elements, especially nitrogen and carbon.

As a first step, a relationship between the gas mass fraction and $Z$ is obtained by solving Eqs. (1) and (2). Their solution depends on the adopted assumptions for $\psi(t), W(t)$ and $A(t)$. In principle, these assumptions are arbitrary and, therefore, the number of models is infinite. Here, two distinct families of 
models refered to as I and II will be considered. Models of class I are those for which the mass of gas, $m_{\mathrm{g}}$, and of stars, $m_{\mathrm{s}}$, within a certain galaxy evolve with time following $m_{\mathrm{g}}=\alpha m_{\mathrm{s}}^{\gamma}$, with $\alpha>0$ and $\gamma$ arbitrary. In the second set of models the star formation rate depends exclusively on $m_{\mathrm{g}}$ according to a law of the form $\psi=\beta m_{\mathrm{g}}^{\varphi}$, with $\beta$ and $\varphi$ either positive or zero. The complete set of both models covers a broad variety of physical situations (including the closed-box model) and it is not difficult to find physical motivations for each of them (except perhaps for those models with $\gamma>2$ ).

These models assume implicitly that the star formation is rather continuous and extended in time. Observations suggest that this property is very representative of dwarf irregular galaxies (e.g. Greggio et al. 1993; Dohm-Palmer et al. 1998; Aparicio \& Tikhonov 2000). Since the last assumption could be inadequate, at least, for bursting galaxies, our study refers only to dwarf, non-bursting, irregular galaxies, as mentioned above.

In a real situation $\gamma$ is expected to fluctuate over time. However, we will assume, as a first approach, that $\gamma$ is constant over time. Varying $\gamma$ in models of type I permits us to consider different scenarios for the temporal evolution of the content of gas and stars in a galaxy. Galaxies with no gas accretion should present $\gamma<0$ as $m_{\mathrm{g}}$ can only decrease with time. Positive values of $\gamma$ are possible if accretion of mass is not negligible. The linear correlation between luminosity and gas content found in dwarf galaxies (see Sect. 2.2) suggests that $\gamma=1$ is possible, although it is not a requirement. We argue that $0<\gamma<1$ may correspond to an effective description of a situation where the mass of gas increases due to accretion but not as fast as the stellar mass does. One would expect $\gamma \approx 1$ if a disk-like component was forming inside-out, keeping the ratio $m_{\mathrm{g}} / m_{\mathrm{s}}$ approximately constant as the disk grows. This is reasonable if, for instance, newly-accreted gas has a higher angular momentum. On the other hand, $\gamma \approx 0$ corresponds to the case of constant mass of gas. The latter case is physically meaningful if infalls and outflows of gas occur simultaneously and they are in rough balance with the star formation rate. According to recent models on the formation and evolution of galaxies (including dwarfs) with star formation feedback and self-regulation (e.g. Efstathiou 2000), it does not seem unrealistic to assume that the mass of gas within a particular galaxy is approximately constant in time. In fact, an enhancement in the infall rate would produce larger perturbations, probably promoting gravitational instabilities and inducing star formation activity. Newly-formed massive stars and supernova explosions could power the outflow of the gas, thus increasing the gas loss. This self-regulated mechanism may result in a relatively small variation of the gas content along the galaxy lifetime (see Efstathiou 2000 for more details).

The second class of models embodies the observational law that the star formation rate follows the surface density to some power (e.g. Kennicutt 1983, 1989, 1998; Martin \& Kennicutt 2001). In this case, a threshold in the gas density is required for triggering star formation.

The number of free parameters is apparently so huge that different situations may arise. Ideally, we would like to make comparisons with observations in order to constrain the admissible values for the different parameters. In the unlikely situation that all the models were not very sensitive to reasonable variations of the parameters we should conclude that the $Z-L$ relationship should be universal and independent of the details of galaxy formation and evolution. However, if there exists a tight empirical relation between $Z$ and $L$ and the models predict a significant scatter, the interesting situation that some kind of self-regulating mechanisms must be at play is promising.

\subsubsection{Models of type I}

For models of type I, Eqs. (1) and (2) can be written in terms of the $v$ parameter defined as $v(t)=m_{\mathrm{s}} / m_{\mathrm{g}}$ (Tinsley 1980). The requirement that there is ongoing star formation in these galaxies imposes the following constraints on the sign of the net rate of inflow $A-W$. When the accretion of gas is larger than the outflows $(A>W), v$ is restricted to values $v>-\gamma$, whereas if the accretion is smaller than the winds, then $v<-\gamma$. Thus, $\gamma \geq 0$ always implies $A>W$. However, for $\gamma<0$, this model allows more loss of mass than accretion, whenever $m_{\mathrm{s}}<(\alpha|\gamma|)^{1 /(1-\gamma)}$.

After some algebra similar to that described in the Appendix A for $\gamma=0$, the equation relating the evolution of the metallicity of a galaxy to its gas mass fraction is, for $\gamma \neq 1$

$\frac{\mathrm{d} Z}{\mathrm{~d} v}=\frac{1}{1-\gamma}\left[y_{z}+\left(Z^{\prime}-Z\right) b_{v} \eta\right]$

with $\eta \equiv 1+\gamma / v$ and $b_{v} \equiv A /(A-W)$. Here $b_{v}, A, W$ and $Z$ depend on $v$ and implicitly on time through $v(t)$.

An infinite variety of models can be constructed by assuming different functions for $b_{v}$. We have explored two different possibilities that can be representative of the evolution of nonstarbursting galaxies. First, it may be interesting to assume that the dimensionless parameter $b_{v}$ is fairly constant in time as a consequence of some self-regulating mechanisms operating between the disk and the halo. We will drop the subscript $v$ to indicate that it is constant, i.e. $b_{v} \equiv b$. We refer to these models as self-regulated models (hereafter SR models). However, this assumption seems to be rather arbitrary. In fact, as suggested by a referee, the gravitational potential due to dark and baryonic matter can determine gas density, the star formation rate and galactic wind, resulting in a coupling between $b$ and $m_{\mathrm{g}}$. In order to know its relevance, a second possibility assuming a dependence of the wind on the star formation rate has been explored. For simplicity, a linear relation $W=\lambda \psi$ was adopted, with $\lambda$ a positive number. We will refer to them as $\lambda$-models of type I. It can be seen that in the latter case, $b_{v} \eta=\eta+\lambda /(1-R)$.

There are some trivial solutions for models I with $\gamma \geq 0$. For such a case and if the rate of infall of gas is zero $(A=0)$, or if outflows and infalls are in balance $(A=W)$, it holds $\psi=0$.

If both infall and outflows of gas are present, and assuming that $b_{v}$ is independent of $v$, the solution of Eq. (3) with $\gamma=0$ is as follows:

$Z(v)=\left(Z^{\prime}+\frac{y_{z}}{b}\right)(1-\exp (-b v))$

Here, the initial metallicity of the galaxy was assumed to be primordial. Since the star formation rate $\psi=(A-W) /(1-R)$ 
(for the case $\gamma=0$ ), the assumption $b=$ const. in Eq. (4) does not necessarily imply that star formation is constant in time. In particular, for $A$ any arbitrary function of time and under the assumption that $W=\lambda \psi$, we have $b=1+\lambda /(1-R)$. For a formal solution in a more general case of $b_{v}(v)$ and other considerations on the resolution of the equation with $\gamma=0$, the reader is referred to Appendix A.

Finally, for $\gamma=1$ and $b_{v}$ independent of $v$, the solution of Eqs. (1) and (2) can be expressed as:

$Z(v)=Z^{\prime}+\left(\frac{y_{z}}{b}\right)\left(\frac{v}{v+1}\right)$

where we have assumed that the metallicity of the gas is primordial at $v=0$.

Solutions for models with $\gamma \neq 0,1$ are given in Sect. 4 .

\subsubsection{Models of type II}

For models of type II, where $\psi=\beta m_{\mathrm{g}}^{\varphi}$, there is no restriction on the sign of the quantity $A-W$. In particular, the cases in which outflows and inflows are in balance $(A=W)$ are also of relevance, and the closed-box model corresponds to $A=W=0$, regardless of the value of $\varphi$. Analogously to type I models, we distinguish between SR models $\left(b_{v}\right.$ constant $)$ and $\lambda$-models $(W=\lambda \psi)$.

The differential equation for the metallicity in models II is expressed in terms of $m_{\mathrm{g}}$ as follows:

$m_{\mathrm{g}} \frac{\mathrm{d} Z}{\mathrm{~d} m_{\mathrm{g}}}=\frac{y_{z} \beta^{\prime} m_{\mathrm{g}}^{\varphi}+\left(Z^{\prime}-Z\right) A}{-\beta^{\prime} m_{\mathrm{g}}^{\varphi}+A-W}$

where $\beta^{\prime}$ is defined as $\beta^{\prime}=(1-R) \beta$.

Due to the lack of any additional information, we have carried out the integration of Eq. (6) assuming that the accretion rate $A$ is constant in time for a particular galaxy.

\subsection{The relationship between metallicity and luminosity}

It can be seen that for most of the models, the relationship between $Z$ and $v$ inferred from Eqs. (3) and (6) is not linear, and for some of the models it saturates exponentially (see, e.g., Eq. (4)). The implications of this result will be discussed more extensively below. Non-linear relationships between $Z$ and $v$ were also found by Gallagher \& Hunter (1982), Matteucci \& Chiosi (1983) and Pilyugin \& Ferrini (2000a). In particular, Pilyugin \& Ferrini presented a logarithm relationship between $Z$ and the gas mass fraction, $\mu$, but then they did obtain a linear empirical correlation between the metallicity and the luminosity. An elaborated model describing the evolution of dIs was proposed by Ferrara \& Tolstoy (2000) for gas-dominated galaxies, neglecting gas accretion. They are particularly interested in the effect the dark matter gravitational potential may have on the gas removal and on the star formation rate. Interestingly, a tight correlation was found between metallicity and dark matter content. A discussion on their approximations and results can be found in Appendix B. The study presented here takes into account accretion of gas and is aimed to include those galaxies that cannot be considered as gas-dominated.
Integration of the differential Eq. (3) for models I and of Eq. (6) for models II, gives the dependence of $Z$ as a function of $v$. However, in order to obtain a relationship between metallicity and luminosity, it is necessary to know the dependence of $v$ as a function of the luminosity which is not free from uncertainties, since $q \equiv m_{\mathrm{s}} / L$ may vary from galaxy to galaxy. This parameter can vary according to the strength of the current star formation event. For the dI galaxies of the Local Group, a nearly-constant ratio is obtained, although the sample was very small (Mateo 1998).

Here the following approach is proposed in order to compare the predictions of the models with the observed $Z-L$ relationship of dwarf irregulars. Those galaxies for which the mass of gas versus luminosity is $m_{\mathrm{g}}=a L+c$, with $a$ and $c$ constant, should follow a particular $Z-L$ relationship. For instance, for the SR model with $\gamma=0$ and given $b$, the $Z-L$ curve would be described by:

$Z=\left(\frac{y_{z}}{b}+Z^{\prime}\right)\left[1-\exp \left(-\frac{q b L}{a L+c}\right)\right]$.

Since we are imposing a relation between $m_{\mathrm{g}}$ and $L$, the curves $Z$ versus $L$ should not be interpreted as evolutionary curves in the sense that a galaxy will not evolve in time following a single curve. The curves describe "snapshots" of the present state of evolution. In practice, only one set of parameters $(a, c)$ fits well most of the galaxies of the sample.

In fact, there exists a general linear trend between luminosity and mass of gas for all the galaxies catalogued as nearby dI (Paper I). The total mass of gas, $m_{\mathrm{g}}$, including atomic and molecular hydrogen as well as $\mathrm{He}$, is assumed to be roughly $1.4 m_{\mathrm{HI}}$. The mass of gas as a function of stellar luminosity for these galaxies is presented in Fig. 1. For comparison, a small subsample of Magellanic irregulars was also included. The masses of atomic hydrogen $\left(m_{\mathrm{HI}}\right)$ and stellar luminosities $\left(L_{B}\right)$ have been compiled from Huchtmeier \& Richter (1988), except the luminosity of NGC 55 which was taken from Puche et al. (1991); they are presented in Table 1. No uncertainties in the data on the $m_{\mathrm{HI}}$ or $L_{B}$ have been found in the mentioned references. The relationship between the mass of gas and the luminosity could be considered linear, with $m_{\mathrm{g}}=0.95 L_{B}+1.4$, where $m_{\mathrm{g}}$ is in units of $10^{8} M_{\odot}$ and $L_{B}$ in units of $10^{8} L_{\odot}$, and a regression coefficient of 0.85 when galaxies with $L_{B}>$ $10^{8} M_{\odot}$ in Table 1 are considered. The most discordant object are the well known dI galaxies DDO 154, UGC 6451 and NGC 1569. This last galaxy is in a post-eruptive phase according to Gónzalez-Delgado et al. (1997) and, therefore, it is expected to deviate from the rest of the galaxies in the $Z-L$ relationship. DDO 154 could also deviate from the general trend since it is a dark-matter dominated galaxy and its associated parameters (e.g. $b$, etc.) may be very different from those of the other galaxies. If these two galaxies are not included, the regression coefficient increases up to 0.95 .

We note that the exact value of $c$ is uncertain, it being any value between 0 and 2 (in units of $10^{8} M_{\odot}$ ). Therefore, for those galaxies with $L_{B}<c \approx 1.4 \times 10^{8} L_{\odot}$, for which the exact value of $c$ is relevant, our predicted $Z-L$ relation should not be compared directly with the data-points, but requires a more careful (point-to-point) analysis. For instance, in the limit case 
$c=0$, the SR model with $\gamma=0$ and fixed $b$ would predict that there should not exist a relationship between metallicity and luminosity. In general, for values of $c$ lower than the value adopted here, $c<1.4$, the region where $Z$ increases linearly with $L$ becomes more narrow, masking even more any trend in the $Z-L$ diagram.

\section{The observational data}

In this section the observational data is presented. Stellar luminosities were taken from Huchtmeier \& Richter (1988), whereas oxygen abundances, based on spectroscopy, were derived by the authors in most of the cases.

The $Z-L$ relationship is commonly presented in terms of the logarithm of the oxygen abundance versus the absolute magnitude in the $B$-band $\left(M_{\mathrm{b}}\right)$. As discussed in Paper $\mathrm{I}$, the $V$ band is a better describer of the typical stellar luminosity of a galaxy, whereas the luminosity in the $B$ band is associated with the luminosity of the recently born stars. To facilitate comparison with previous works, we will use also $L_{B}$ throughout the paper. However, we prefer to study the relation between $Z$ and $L$ instead of the familiar plot $12+\log (\mathrm{O} / \mathrm{H})$ vs. $M_{\mathrm{b}}$. Although the use of $\mathrm{O} / \mathrm{H}$ is not so rare in the literature (see, e.g. González-Riestra et al. 1988; Walsh \& Roy 1989, 1993; Skillman \& Kennicutt 1993; Chandar et al. 2000), the real reasons for this choice and other subtleties will become clear in Sect. 6.2.

\subsection{The metallicity data}

A subsample of 16 galaxies from Table 1 was observed at different campaigns in order to obtain their metal content. Extensive results are published elsewhere (Hidalgo-Gámez 1999; Hidalgo-Gámez et al. 2001a,b; Hidalgo-Gámez \& Olofsson 2002) but only the oxygen abundances are presented here. For various reasons, oxygen is the representative element of the chemical abundances when gasrich galaxies are studied (Osterbrock 1989). In Paper I it was argued that nitrogen is a more favorable element for comparing with the luminosity of the stellar content, but the main advantage of oxygen are the relatively small uncertainties which appear to be crucial in this study.

As discussed in Paper I, the metallicity was determined by means of the temperature-sensitive method based on the intensity of the [OIII] $] 4363 \AA$ emission line (Osterbrock 1989). Another way to proceed in order to determine the abundance of oxygen, if the line [OIII] $\lambda 4363 \AA$ is absent or too weak in the spectrum, is to use indirect methods. They are either theoretical (Olofsson 1997), semi-empirical (Pagel et al. 1979; McGaugh 1994), or empirical (Skillman 1989). For comparison, the oxygen abundance was determined for all the galaxies also using these various indirect methods. Comparing the values obtained with the semi-empirical and temperature sensitive methods, good agreement was found close to the region of $12+\log (\mathrm{O} / \mathrm{H}) \sim 8.1$. At lower and higher abundances large deviations became evident. The obtained differences were as large as 0.5 dex, especially for low-metallicity galaxies. When the theoretical method is used, the difference in the oxygen

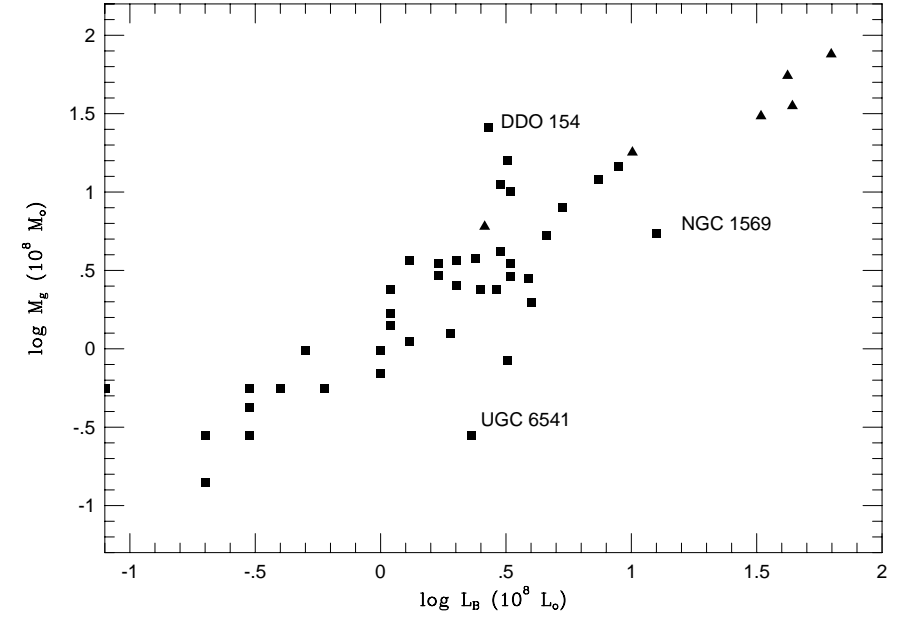

Fig. 1. Mass of gas versus $L_{B}$ for the nearby dI galaxies (filled squares). Seven Magellanic irregulars (filled triangles) have been added for comparison. Data are from Huchtmeier \& Richter (1988), except for NGC 55 whose luminosity was taken from Puche et al. (1991). The total mass of gas is $1.4 m_{\mathrm{HI}}$. The regression coefficient of the total sample is $r_{l}=0.85$.

abundances is smaller, that is no more than 0.2 dex, but still unsatisfactory. It should be noted that in the study by Olofsson (1997), the calibration was aimed at galaxies undergoing intense star formation, such as blue compact galaxies. To summarize, all of the available indirect methods introduce uncertainties of unknown quantities in the derivation of the oxygen abundance so that the ideal situation is to use the temperaturesensitive method.

As a result of this requirement, data on five of those galaxies were not included in this investigation (DDO 154, DDO 69, DDO 70, DDO 75 and ESO 594-G004). To increase the sample, data on two more dIs and the non-dI (or Magellanic irregular) galaxies presented in Table 4 of Paper I were added. Their oxygen abundances were obtained from the literature (see Table 2 for references). The uncertainties of the data obtained by the authors were under control because they were reduced and analyzed in a similar way, but the data from the literature probably adds an unknown amount of scatter to the diagram due to unknown uncertainties.

\subsection{The observational relationship}

The oxygen abundances and stellar luminosities for all the galaxies under study are presented in Table 2. Figure 2a shows the bona fide $Z-L$ relationship for both dwarf and Magellanic irregular galaxies. Several features deserve a detailed explanation. First, it is evident that more observational data on dwarf galaxies with luminosities larger than $20 \times 10^{8} L_{\odot}$ and oxygen abundance $\mathrm{O} / \mathrm{H} \sim 1.6 \times 10^{-4}$ are needed in order to fix the shape of the relationship at high luminosities, but notice that they are probably at the limit of being dwarf galaxies themselves (see Paper I). Similarly, it is also important to have good determinations of the metallicity for very low-luminosity galaxies. Both DDO 167 and DDO 155 are particularly interesting. They have $L_{B} \approx 0.2 \times 10^{8} L_{\odot}$ and relatively low oxygen abundances $\left(\mathrm{O} / \mathrm{H}=1.58 \times 10^{-5}\right.$ and $2.5 \times 10^{-5}$, respectively) but, 
Table 1. H I mass data and the luminosity in the blue band for the nearby dIs and a subsample of non-dI galaxies. Column 1 lists the identification of the galaxy, the mass of $\mathrm{H}_{\mathrm{I}}$ is given in Col. 2 and $L_{B}$ is presented in Col. 3, both in units of $10^{8}$ solar values. The ratio between these two parameters is presented in Col. 4 . The last column shows the $L_{B} / m_{\mathrm{g}}$ ratio, with $m_{\mathrm{g}}=1.4 m_{\mathrm{HI}}$. All data are from Huchtmeier \& Richter (1988), except the luminosity of NGC 55 which is taken from Puche et al. (1991).

\begin{tabular}{cccccccccc}
\hline \hline Galaxy & $m_{\mathrm{HI}}$ & $L_{B}$ & $L_{B} / m_{\mathrm{HI}}$ & $L_{B} / m_{g}$ & Galaxy & $m_{\mathrm{HI}}$ & $L_{B}$ & $L_{B} / m_{\mathrm{HI}}$ & $L_{B} / m_{g}$ \\
\hline IC 10 & 3.8 & 4.6 & 1.20 & 0.86 & DDO 155 (Gr 8) & 0.2 & 0.2 & 1.0 & 0.71 \\
DDO 226 & 0.2 & 0.3 & 1.49 & 1.07 & DDO 165 & 0.9 & 1.9 & 2.13 & 1.51 \\
DDO 6 & 0.1 & 0.2 & 2 & 1.43 & UGC 8215 & 0.4 & 0.08 & 2 & 1.43 \\
IC 1613 & 0.8 & 1.3 & 1.61 & 1.16 & DDO 167 & 0.3 & 0.3 & 1.0 & 0.71 \\
ESO 245-G05 & 7.2 & 3.3 & 0.46 & 0.33 & DDO 168 & 5.7 & 5.3 & 0.93 & 0.66 \\
UGCA 105 & 11.4 & 3.2 & 0.25 & 0.20 & DDO 169 & 1.8 & 2.0 & 1.10 & 0.79 \\
NGC 1569 & 3.9 & 12.6 & 3.23 & 2.31 & ESO 324-G024 & 8.0 & 3.0 & 0.37 & 0.27 \\
NGC 2366 & 8.6 & 7.4 & 0.86 & 0.61 & UGC 8508 & 2.1 & 1.7 & 0.81 & 0.58 \\
DDO 47 & 2.6 & 1.3 & 1 & 0.37 & DDO 181 & 1.0 & 1.1 & 1.10 & 0.79 \\
UGC 4115 & 1.2 & 1.1 & 0.92 & 0.65 & DDO 183 & 0.7 & 1.0 & 1.43 & 1.02 \\
DDO 50 (Ho II) & 10.4 & 8.9 & 0.86 & 0.61 & DDO 187 & 0.4 & 0.4 & 1.00 & 0.71 \\
DDO 53 & 0.7 & 0.5 & 0.71 & 0.51 & DDO 190 & 1.7 & 2.5 & 1.47 & 1.05 \\
DDO 63 (Ho I) & 1.4 & 4.0 & 2.86 & 2.04 & IC 4662 & 1.7 & 2.9 & 1.72 & 1.22 \\
DDO 69 (Le A) & 0.4 & 0.3 & 0.75 & 0.54 & ESO 594-G004 (Sag Dig) & 0.08 & 0.02 & 0.25 & 0.18 \\
DDO 70 (Sex A) & 1.7 & 2.5 & 1.47 & 1.05 & NGC 6822 & 2.6 & 2.0 & 0.77 & 0.55 \\
DDO 75 (Sex B) & 2.5 & 1.7 & 0.68 & 0.49 & DDO 210 & 0.07 & 0.07 & 1.00 & 0.71 \\
UGC 6541 (Mrk 178) & 0.2 & 2.3 & 1.49 & 1.07 & IC 5152 & 0.5 & 1.0 & 2 & 1.42 \\
DDO 99 & 2.7 & 2.4 & 0.89 & 0.63 & DDO 216 (Pegasus) & 0.06 & 0.002 & 3.33 & 2.38 \\
NGC 4068 & 2.5 & 3.3 & 1.32 & 0.94 & DDO 221 & 1.8 & 2.0 & 1.10 & 0.79 \\
NGC 4163 & 0.6 & 3.2 & 5.26 & 3.81 & NGC 55 & 39.4 & 42.0 & 1.07 & 0.76 \\
DDO 126 & 1.7 & 1.1 & 0.65 & 0.46 & NGC 4214 & 25.3 & 43.9 & 1.73 & 1.24 \\
DDO 125 & 2.0 & 3.9 & 1.92 & 1.39 & NGC 4449 & 54.1 & 62.7 & 1.16 & 0.83 \\
UGC 7605 & 0.4 & 0.6 & 1.49 & 1.07 & ESO 381-G20 & 4.3 & 2.6 & 0.60 & 0.43 \\
DDO 133 & 3.0 & 3.0 & 1.00 & 0.71 & IC 2574 & 12.8 & 10.1 & 0.79 & 0.56 \\
DDO 141 & 2.9 & 3.3 & 1.14 & 0.81 & NGC 4395 & 21.8 & 32.9 & 1.51 & 1.08 \\
DDO 154 & 18.4 & 2.7 & 0.15 & 0.10 & NGC 5408 & 7.2 & 2.2 & 0.30 & 0.22 \\
\hline
\end{tabular}

unfortunately, with high uncertainties $\left(2 \times 10^{-5}\right.$ and $0.8 \times 10^{-5}$, respectively).

Figure $2 \mathrm{~b}$ shows an enlargement of luminosities lower than $20 \times 10^{8} L_{\odot}$ for higher resolution. For these galaxies there is no correlation the regression coefficient is very weak $\left(r_{l}=0.4\right)$ and the scatter is very large. This confirms previous claims that the traditional $Z-L$ correlation gets weaker at low metallicities and low luminosities (RM; Skillman 1999). Moreover, since the metallicities for the three galaxies with lower $Z$ and $L$ present large uncertainties, their real values could be somewhat different, as claimed by Moles et al. (1992) and SKH. If these galaxies are more metallic, even a weak anticorrelation might appear.

\section{Comparing models and data}

In order to compare the predictions of the models with observations we need to define the range of validity of the free parameters of the models and to fix the value of $y_{z}$ and $R$. The yield, $y_{z}$, is always very difficult to determine empirically. It depends on the IMF and on the stellar nucleosynthesis (Lequeux et al. 1979). When a stellar model is chosen (mainly by fixing the mass loss and the IMF parameters), the value of the theoretical yield is not expected to vary. A standard net yield for the interstellar abundance of oxygen is $y_{\mathrm{o}}=0.004$ (Maeder 1993) and it is used here. A typical value of $R=0.6$ was adopted from Maeder (1993).

Typical values for $q$ can be found in Pilyugin \& Ferrini (2000a). Fitting observational data with their model they obtained that $q$ ranges between 0.27 and 0.84 . The value of $q$ may vary with time for a particular galaxy, decreasing when it experiences an event of star formation due to the luminosity enhancement.

It has to be reminded that there still exist free parameters; for SR models of type I these are $(\gamma, b)$ and for SR models of type II $(\varphi, \beta, b)$, whereas for $\lambda$-models of type I and of type II they are $(\gamma, \lambda)$ and $(\varphi, \beta, \lambda)$, respectively. For models of type II we use units such as the initial mass of gas is 1 and $A=1$. Although the values of $b$ and $\lambda$ are very uncertain since there is little observational information on the amount of infall or outflow gas in galaxies, large values of $b$ and $\lambda$ are unsatisfactory. For instance, values of $b>20$ would imply a fine-tuning between $A$ and $W, A / W \sim 1.05$. The value of $\beta$ determines the variations in the mass of gas during the lifetime of the galaxy. Since for the galaxies under study $v \leq 3$, extreme variations in the mass of gas are unlikely to occur as it would imply either an extremely large accretion relative to the average star formation rate for $A>W$, or an unacceptably intense wind for $W>A$. This fact constrains the admissible values for $\beta$. 
Table 2. Luminosities and abundances for the dI sample. The galaxy identification is given in Col. 1 . Column 2 lists $L_{B}$, taken from Huchtmeier \& Richter (1988). Column 3 shows the oxygen abundance, and its uncertainties, when available, are given in Col. 4. References for the metallicity data are presented in Col. 5. 1) Hidalgo-Gámez et al. (2001a); 2) Hidalgo-Gámez et al. (2001b); 3) Hidalgo-Gámez \& Olofsson (2002); 4) Hidalgo-Gámez (1999); 5) González-Delgado et al. (1994); 6) Devost et al. (1997); 7) Vigroux et al. (1987); 8) SKH; 9) Webster et al. (1983); 10) Kobulnicky \& Skillman (1996); 11) McCall et al. (1985); 12) Masegosa et al. (1994); 13) Masegosa et al. (1991).

\begin{tabular}{ccccc}
\hline \hline Galaxy & $L_{B}$ & $\mathrm{O} / \mathrm{H}$ & $\Delta(\mathrm{O} / \mathrm{H})$ & Ref. \\
\hline IC 10 No. 45 & $\left(10^{8} L_{\odot}\right)$ & $\left(10^{-4}\right)$ & $\left(10^{-4}\right)$ & \\
ESO 245-G05 No. 19 & 3.6 & 1.00 & 0.07 & 4 \\
ESO 245-G05 No. 12 & 3.3 & 0.63 & 0.01 & 2 \\
DDO 50 (Ho II) & 8.5 & 0.63 & 0.06 & 4 \\
DDO 53 & 0.5 & 0.32 & 0.06 & 4 \\
DDO 155 (Gr 8) & 0.2 & 0.25 & 0.08 & 3 \\
DDO 167 & 0.3 & 0.16 & 0.2 & 3 \\
DDO 168 & 5.3 & 0.32 & 0.2 & 3 \\
DDO 190 & 2.5 & 1.00 & 0.03 & 3 \\
IC 4662 A1/A2 & 2.9 & 1.48 & 0.03 & 2 \\
IC 4662 D & 2.9 & 0.5 & 0.1 & 2 \\
NGC 6822 HV & 2.0 & 1.32 & 0.02 & 1 \\
NGC 6822 HX & 2.0 & 1.26 & 0.06 & 1 \\
IC 5152 & 1.0 & 1.58 & 0.1 & 3 \\
NGC 2366 & 7.4 & 0.79 & 0.01 & 5 \\
NGC 1569 & 12.6 & 1.58 & 0.05 & 6 \\
NGC 55 & 42.0 & 2.27 & - & 7 \\
NGC 4214 & 43.9 & 1.66 & 0.02 & 10 \\
NGC 4449 & 62.7 & 1.70 & - & 8 \\
ESO 381-G20 & 2.6 & 0.87 & - & 9 \\
IC 2574 & 10.1 & 0.79 & 0.05 & 13 \\
NGC 4395 & 32.9 & 0.73 & - & 11 \\
NGC 5408 & 2.2 & 1.17 & - & 12 \\
\hline
\end{tabular}

Different $Z-L$ curves predicted by the models are plotted in Figs. 3 to 6 for $Z^{\prime}=0, a=0.95 M_{\odot} / L_{\odot}$ and $c=1.4 \times 10^{8} M_{\odot}$. Our fiducial value for $q$ is 0.5 but curves for $q=0.9$ and $q=0.1$ are also shown in each figure. The curves are presented in the plane $\mathrm{O} / \mathrm{H}$ vs. $\log L_{B}$ to increase the resolution of the data points, especially at low luminosities. It should be kept in mind, however, that since in most of the models $Z$ saturates with $L$, the curves appear much more linear in such a plot than on a linear scale. Integration was performed imposing $Z=0$ at $L=0$, except for models with $\gamma>1$ for which the initial condition was imposed well outside the box shown in Fig. 3, in which case the curve is not sensitive to the exact initial values. Only those curves with metallicities in the range of the observed values were selected. The largest value corresponds
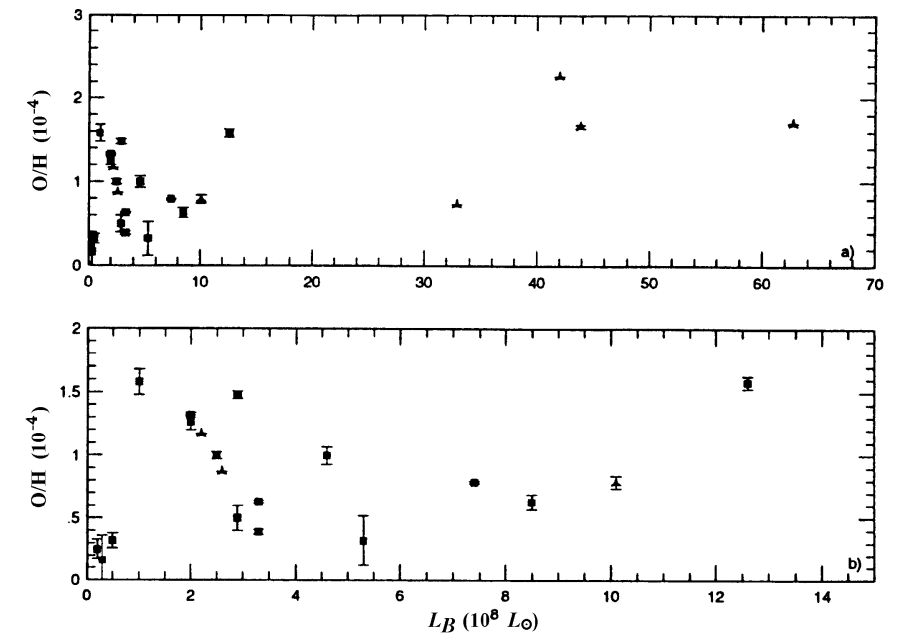

Fig. 2. Panel a) shows the bona fide observational $Z-L$ relationship for the whole sample of irregular galaxies. Panel b) corresponds to a zoom of the inner region in order to increase resolution. The filled squares indicate dIs and the empty triangles are classified as Magellanic Irregulars, designated non-dI in Paper I. Data on the $L_{B}$ and metallicity are from Table 2.

to $\mathrm{LMC}\left(\mathrm{O} / \mathrm{H}=2 \times 10^{-4}\right)$ while the smallest one corresponds to I Zw $18\left(\mathrm{O} / \mathrm{H}=1.5 \times 10^{-5}\right)$. Although these galaxies are not "real" dI, they bracket the metallicity of dwarf irregulars and, as studied in Hidalgo-Gámez \& Olofsson (2002), Magellanic irregulars, blue compacts and dwarf irregulars do not present differences in their metal content. Interestingly, all the models predict a plateau in metallicity at luminosities, on average, $\geq 25 \times 10^{8} L_{\odot}$, although the exact value depends on the model.

In Fig. 3 the $Z-L$ curves are presented for SR type I models with different $\gamma$. When $\gamma \rightarrow 0$ the mass of gas is nearly constant in time, while large values of $\gamma$ indicate that star formation becomes much more inefficient as the galaxy grows. In order to explain galaxies with high metallicities and low luminosities it is necessary to increase $q$ up to 0.9 if $\gamma=0$. For $\gamma \geq 0$ and $q \approx 0.5$, values $b \geq 2$ are required to fit those galaxies with $\mathrm{O} / \mathrm{H} \approx 3-5 \times 10^{-5}$ and $L \approx 5-10 \times 10^{8} L_{\odot}$. The metallicity of these galaxies is preferably reproduced with negative values of $\gamma$ (see Fig. 3).

Type I $\lambda$-models are presented in Fig. 4 with different values of $\gamma$ and $\lambda$. In order to see the influence of the wind, the curves with $\lambda=0.2,1$ and 2 were also drawn. The low luminosity galaxies with high metallicities (e.g. NGC 6822 and IC 5152) still need values of $q \approx 0.9$ and small values of $\lambda$. In fact, the metallicities for these galaxies are so high that any model requires a low wind. The constant star formation rate through time found for NGC 6822, typically $3 \times 10^{-9} M_{\odot} \mathrm{yr}^{-1} \mathrm{pc}^{-2}$ during the last $12-15 \mathrm{Gyr}$ (Wyder 2001), would suggest that $\gamma=0$ or 1 for this galaxy. IC 5152, on the other hand, may have a lower metallicity than the value from Hidalgo-Gámez \& Olofsson (2002) (H. Lee, private communication). In any case, the metallicity of these two galaxies can also be reproduced well with the closed-box model (see Fig. 5).

Type II SR-models are presented in Fig. 5 for different values of $b$. For $b<0$ the smallest values in the metallicity are obtained. Again, the low luminosity galaxies with high 


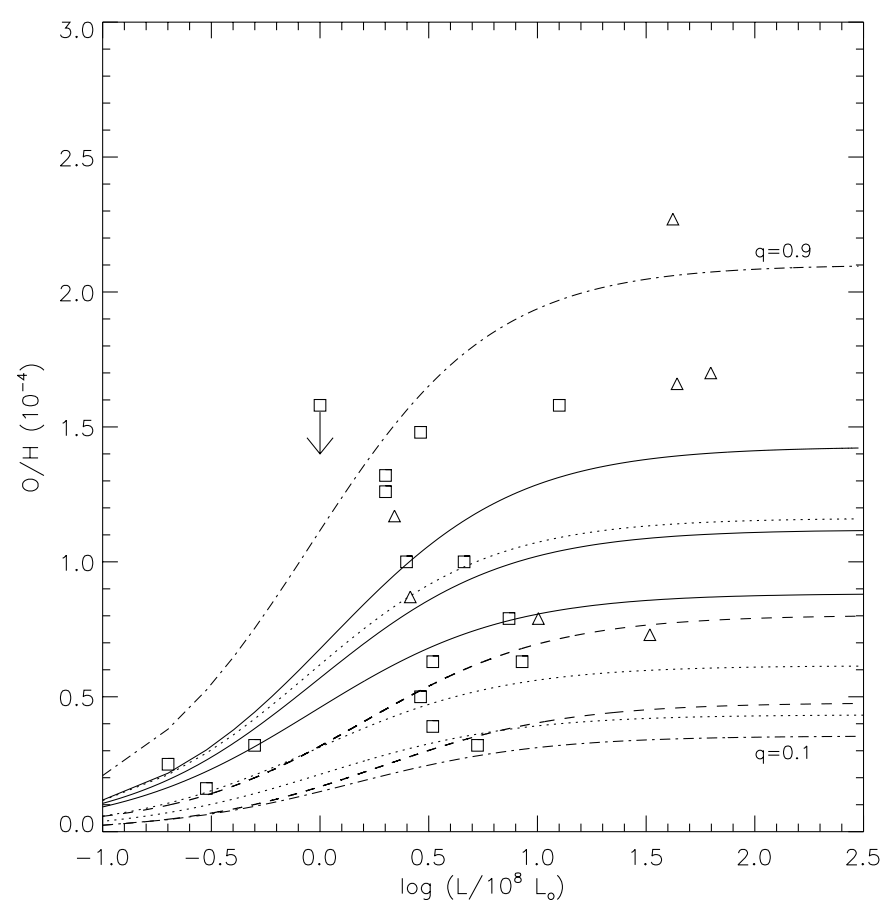

Fig. 3. Theoretical $Z-L$ curves for the SR-model of type I for different values of $\gamma$ and $b$. All the models have $q=0.5$ except those indicated by the dot-dashed lines. From top to bottom: the solid lines represent those models with $b=1.1$ and $\gamma=0,1,2$, respectively, the dotted lines show models with $b=2$ and $\gamma=0,1,2$, and the dashed lines stand for models with $\gamma=-1$ and $b=-0.5,-3$, respectively. Finally, the dot-dashed lines refer to the "standard" model with $\gamma=0, b=1.1$ and $q=0.9$ (upper curve) and $q=0.1$ (lower curve). The observational data points are also plotted with the same symbols as in Fig. 1. The downward vertical arrow indicates that the metallicity is an upper limit.

metallicities are fitted when $b, \beta$ and $q$ are allowed to vary. Finally, curves for type II $\lambda$-models with different values of $\lambda$ and $\varphi$ are plotted in Fig. 6. The value of $\beta$ was fixed to 0.3 because, in the range of interest, $\lambda$ is the most influential parameter. Furthermore, it can be seen that the curves are not very sensitive to $\varphi$.

There are some universal features that do not depend on the details of the models. First, all the models require an intense wind (either $b$ or $\lambda=2$ ) or low values of $q$ (typically $0.3-0.5$ ) to explain those galaxies with $\mathrm{O} / \mathrm{H} \leq 0.6 \times 10^{-4}$ and luminosities $\approx 4-8 \times 10^{8} L_{\odot}$. These galaxies are the most interesting as they can be explained using different models with different set of parameters. Second, the relationship between $Z$ and $L$ is not linear for a given set of parameters but saturates exponentially, reaching a plateau at relatively low luminosities.

It is known that both the yields and the $q$ ratios will not be the same for all the dwarf galaxies, especially the latter. The main influence of $q$ is the change in the asymptotic value of the metallicity reached at large luminosities, with the lowest values corresponding to the lowest $q$ 's. In principle, blue compact galaxies are expected to have small values of $q$ and, therefore, should have smaller metallicities at a given luminosity. However, for a sample of galaxies containing low surface brightness galaxies, Magellanic irregulars and three blue

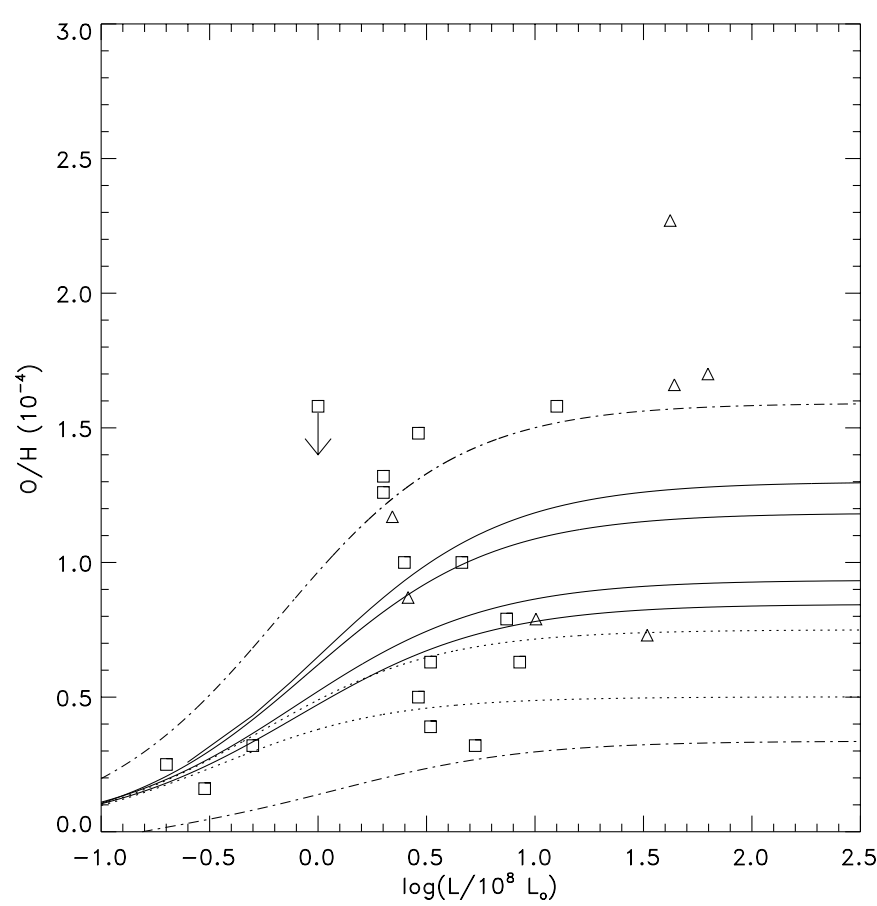

Fig. 4. Theoretical curves for $\lambda$-models of type I, for different $\gamma$ and $\lambda$. Except for the dot-dashed lines, a value of $q=0.5$ was adopted. The solid lines are the predicted $Z-L$ relation for $\lambda=0.2$ and $\gamma=0,0.5,1.5$ and 2, from top to bottom. In order to know the importance of the wind, the upper dotted curve corresponds to $\gamma=0.5$ and $\lambda=1$, whereas the lower dotted curve is for $\gamma=0.5$ and $\lambda=2$. The upper dot-dashed line shows a model with $\gamma=0.5, \lambda=0.2$ but $q=0.9$, while the lower dot-dashed curve is for $\gamma=0.5, \lambda=0.2$ but $q=0.1$ in order to see the importance of variations in $q$. Symbol coding as in Fig. 1.

compact galaxies in addition to the dIs, we do not observe such a feature and, instead, the same values of $q$ match the range of metallicities for each type of galaxies (Hidalgo-Gámez \& Sánchez-Salcedo 2002).

Obviously, the data points are rather dispersed and do not follow a single curve and hence they cannot be fitted with just a single model and a given set of parameters. This should be the case if galaxies do not have any preferent values for these parameters. However, if a self-regulating mechanism between the halo and the disk is operative, it is possible that the parameters are not so different from galaxy to galaxy. In the following we will show that the case of $m_{\mathrm{g}}$ constant (i.e. $\gamma=0$ ) can explain the range of metallicities for a certain $b$, due to variations of $q$. This feature implies that the existence of a self-regulation mechanism cannot be ruled out.

\section{Irregular galaxies with different evolution. The influence of $b$}

In order to gain more insight into the problem, it might be interesting to study in more detail whether variations of only one parameter are enough to explain the observations. If this is the case, some clues about the amount of gas lost or gained by the galaxy could be inferred according to their positions in the diagram. Due to its simplicity, we will focus here on the SR type I 


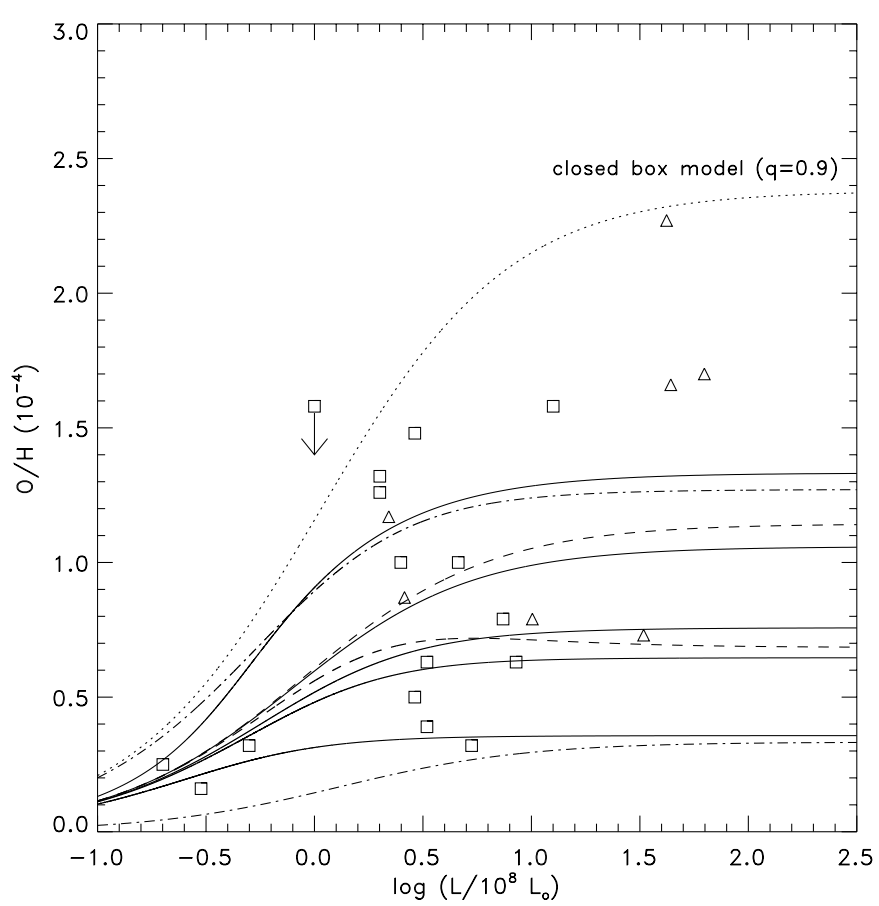

Fig. 5. Theoretical curves for SR-models of type II and different combinations of the parameters. The dotted line corresponds to the closedbox model with $q=0.9$. For the five solid lines $\varphi=1$ and $q=0.5$ but $b$ and $\beta$ are different. From top to bottom they have the following values $(b=1.1, \beta=0.5),(b=-3, \beta=2.5),(b=-3, \beta=1.25)$, $(b=-1.5, \beta=1.25),(b=-0.15, \beta=2.5)$, respectively. The dotdashed lines have $b=-3$ and $\beta=2.5$ but the upper line is for $q=0.9$ whereas the lower lines is for $q=0.1$. In order to see the influence of $\varphi$, curves with $b=-3, \beta=2.5$ and $\varphi=0.3$ (upper dashed line) and $\varphi=3$ (lower dashed line) are displayed together. Symbol coding as in Fig. 1.

model with $\gamma=0$. For this case, the $b$ parameter lies in the range $1<b<\infty$ since the outflow can never be larger than the infall. In the limit $b \rightarrow 1$, the outflows are negligible, while for $b \rightarrow \infty$, outflow and inflow tend to be in balance.

In Fig. 7 the observational data points together with theoretical curves with different values of $q$ and $b$ for this model are presented. We see that most of the metallicities for this sample of galaxies can be explained for $1 \leq b \leq 3$.

Large values of $b$, say $b>10$, imply $W \approx A$, but also a low star formation rate as compared to the accretion rate $(\psi \propto A-W)$. This means that only very low mass galaxies may present high values of $b$, since more massive galaxies cannot energize a wind with such low levels of star formation activity. This might explain the lack of very low metallicity galaxies $\left(\mathrm{O} / \mathrm{H}<3 \times 10^{-5}\right)$

Several outstanding features of this figure deserve explicit comments. Interestingly, most of the metallicities (except perhaps NGC 55) are reproducible with $b \approx 2$, allowing only variations of $q$ between $0.1-0.9$. This fact implies that a selfregulation mechanism able to keep all the galaxies with the same $b$ cannot be ruled out. However, it is also possible that different groups of galaxies may have different $b$. In fact, a group of galaxies are well fitted with $b=1.75$, which corresponds

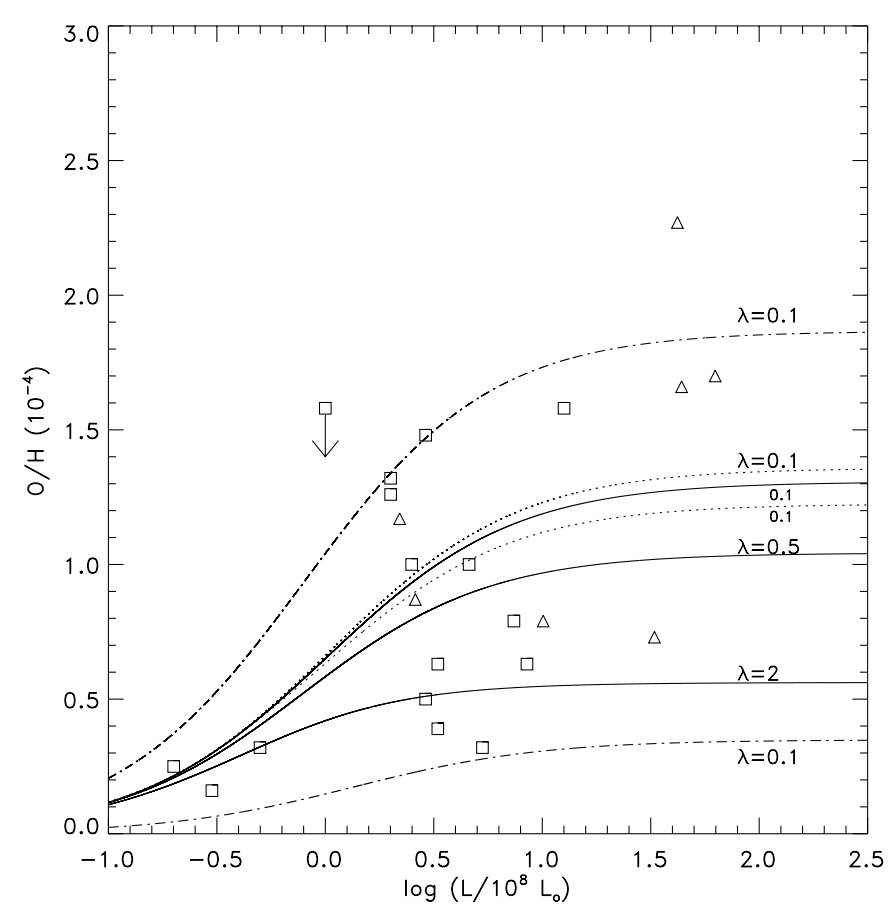

Fig. 6. Theoretical $Z-L$ curves for $\lambda$-models of type II with $\beta=0.3$ (the models are not very sensitive to variations in $\beta$ ). The values of $\lambda$ are indicated for each curve. All the curves have $q=0.5$, except for the upper dot-dashed line $(q=0.9)$ and for the lower dot-dashed one ( $q=0.1$ ). All the curves were calculated for models with $\varphi=1$, except the dotted lines which correspond to $\varphi=3$ (upper dotted line) and $\varphi=0.3$ (lower dotted line). Symbol coding as in Fig. 1.

to $A=2.3 W$, and $q=0.9$. Galaxies illustrative of this group are NGC 4449, NGC 4214 and NGC 1569. A second group of galaxies is fitted well with $b=2.5$ and $q=0.3$. Examples are IC 2574, DDO 50, NGC 2366 and ESO 245-G05. Notice that these sets of parameters are not unique for each of these groups of galaxies. Other values also fit the data points of the second group, e.g. $(b=1.1, q=0.25)$ or $(b=4.5, q=0.5)$. In this sense, each data point is multi-valuated in the $(b, q)$ plane.

Different values of $b$ for these galaxies might be a consequence of different gravitational potentials mainly due to differences in the dark matter component. A first inspection shows that galaxies of the second group are among the largest galaxies of their type. A comparison of both the gas and stellar potential and $v$ was carried out between the seven galaxies. All the galaxies in each group present similar values for $v$, as well as for the gravitational potential of the visible matter. However, remarkable differences between the groups were found, especially for the gravitational potential created by the gas component but also for $v$. Probably, the dark matter halo dominates the gravitational potential and determines the value of $b$.

A more realistic scenario should take into consideration that $b$ may vary in time during the evolution of a galaxy. In fact, two galaxies with the same $v$-averaged value of $b$ may present different metallicities. To quantify this effect, we compare the metallicity for two galaxies with identical $v$-averaged $b=2.5$. Suppose that for the first galaxy, $b=3$ for $0 \leq v<0.5$ and $b=2$ for $0.5 \leq v \leq 1$, whereas for the second galaxy it holds 


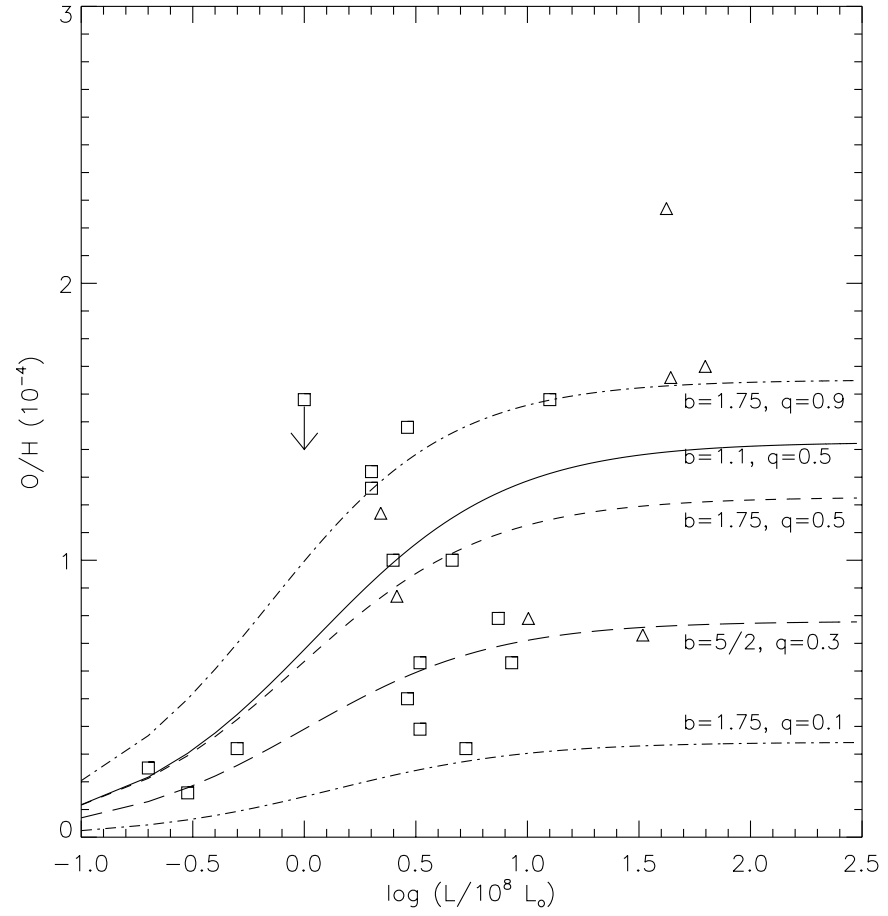

Fig. 7. The fitting of the observational data for SR type I model with $\gamma=0$. Much of the scatter can be explained by varying $q$ between $0.1-0.9$ for $b=1.75$. The galaxy with the highest metallicity corresponds to the magellanic-type NGC 55.

that $b=2$ for $0 \leq v<0.5$ and $b=3$ for $0.5 \leq v \leq 1$. The difference in the metallicity at $v=1$ is $\sim 25 \%$. This corresponds to the maximum scatter in metallicity associated with temporal variations of $b$ in a certain galaxy.

\section{The origin of a linear trend between $\log (\mathrm{O} / \mathrm{H})$ and absolute blue magnitude}

As mentioned in the Introduction, the $Z-L$ relationship for $\mathrm{dI}$ galaxies has been established from three different studies during the last decade or so (SKH, RM and Paper I). Recently, Skillman et al. (2002) have extended the sample by including some dwarf irregular galaxies from the Sculptor Group. It is customary to plot $12+\log (\mathrm{O} / \mathrm{H})$ as a function of $M_{\mathrm{b}}$ instead of $\mathrm{O} / \mathrm{H}$ versus $L_{B}$. The strong linear correlation between $\log (\mathrm{O} / \mathrm{H})$ and $M_{\mathrm{b}}$ found by SKH may have two explanations. Firstly, it is obvious that if the relation between $Z$ and $L$ is approximately linear within a certain range in luminosities, then $12+\log (\mathrm{O} / \mathrm{H})$ versus $M_{\mathrm{b}}$ must be also linear in that range. In the next subsection we study whether this is the case for the mentioned samples. The second possibility is that the dispersion in the free parameters (e.g. $b, \gamma$ and so on) plus a poor sampling produce a linear $\log -\log$ appearance (Sect. 6.2).

\subsection{Comparison between different samples. The abundance of gas-rich galaxies}

SKH obtained a very strong correlation which has been getting weaker over the time. In particular, the large differences

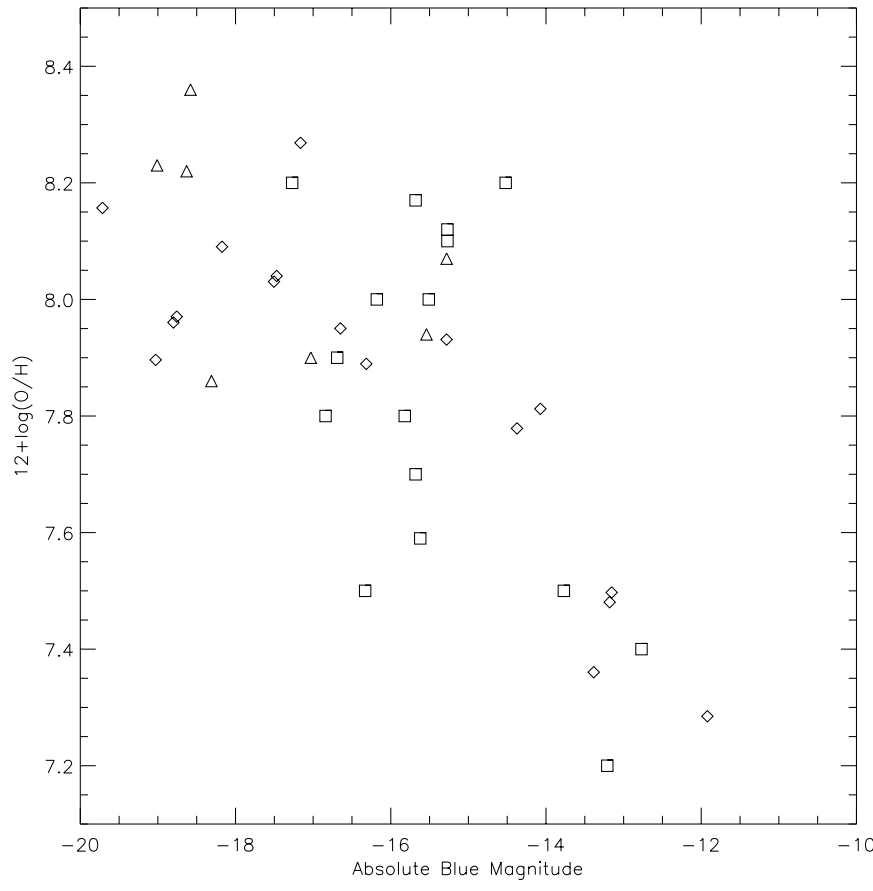

Fig. 8. Synthetic $\log (\mathrm{O} / \mathrm{H})$ versus absolute blue magnitude for a realization of 20 galaxies following a model with $m_{\mathrm{g}}$ constant (diamonds) together with data-points (squares and triangles). The values of $M_{\mathrm{b}}, b$ and $q$ were drawn randomly from -20 to $-10,1$ to 3 and 0.2 to 0.95 , respectively. The existence of a linear trend remarkably similar to the data-points but with high scatter is apparent in this realization.

between the $Z-L$ correlations obtained by SKH and Paper I are quite distressing. One argument favoring the tight linear correlation by SKH could be that the galaxies in their sample are very gas rich. If the gas mass is much larger than the stellar mass, $v$ is small and a linear correlation will emerge between $Z$ and $v$ after a Taylor expansion. This condition is $q b L /(a L+c) \ll 1$, i.e. galaxies with extremely low luminosities. On the contrary, for luminosities $L \geq c /(q b-a)$ that occur when the stellar mass is comparable to or larger than the gas mass, such expansion cannot be applied and the relationship is not linear. In this sense, it is important to check whether the dwarf galaxies are real gas-rich galaxies, or both stellar and gas mass are comparable. The ratio $m_{\mathrm{s}} / m_{\mathrm{g}}$ is equivalent to $q L_{B} /\left(1.4 m_{\mathrm{HI}}\right)$. In the following we will use the ratio $L_{B} / m_{\mathrm{g}}$ as the indicative parameter, but it should kept in mind that $L_{B}$ only reflects the present star formation, and tells us nothing about the old stellar population. Therefore, the ratio $L_{B} / m_{\mathrm{g}}$ does not correspond exactly to the stellar fraction. However, a low value of this ratio can be indicative of a large gas fraction.

From the data of Table 1 it is clear that only four objects show $L_{B} / m_{\mathrm{g}}$ values larger than 2 . On the other hand, 16 out of 45 galaxies present $L_{B} / m_{\mathrm{g}}$ smaller than 0.6 and, therefore, are dominated by the gaseous component. For the remaining 25 galaxies, the stellar mass is of the same order or larger than the mass of gas. In the subsample of galaxies given in Table 2, 13 out of 20 galaxies have comparable mass in gas as in stars. In the light of these data, a model on dwarf galaxies 
which does not consider stellar mass could not be applied to the majority of dwarf irregular galaxies.

The investigation carried out by van Zee et al. (1997) for a sample of low surface brightness dwarf galaxies favors this argument. These galaxies are claimed to be gas-rich, with $L_{B} / m_{\mathrm{HI}}$ ratios smaller than 0.5 for $80 \%$ of the galaxies. They found a good correlation between the oxygen abundance and the absolute magnitude. The weak point in their study is that the chemical abundances of half of their sample are not obtained with the temperature sensitive method, that, as previously discussed, could introduce some unknown uncertainties.

An inspection of Table 1 will shed light on the differences, if any, between the three samples of galaxies used by SKH, RM and Paper I. 35\% of the galaxies studied by SKH are dominated by gas $\left(L_{B} / m_{\mathrm{g}}<0.6\right), 29 \%$ in the RM sample and $40 \%$ in Paper I. Moreover, the three samples have a total of 10 galaxies in common, four of them gas-dominated. If the fraction of gasrich galaxies is the key factor, the relationships in SKH and Paper I should be very similar due to the similar percentage of gas-rich galaxies in both samples. Since this is not the case, it is concluded that the differences between the empirical $Z-L$ relationships are not due to differences in the gas content of their galaxies.

Recently, Pilyugin (2001) obtained a linear correlation between the oxygen abundance and the absolute magnitude for a sample of dI galaxies. He used a method based on the strength of the forbidden oxygen lines [OIII] $\lambda 5007 \AA$ and [OII] $\lambda 3727 \AA$. He concluded that the weakness of the relationships found in RM and in Paper I is caused by the large uncertainties associated with the standard method. As discussed in Sect. 3.1, remarkable differences are found for the oxygen abundance with the standard and the semi- or empirical methods. The difference is 0.16 for DDO 168, 0.21 for DDO 155 and 0.56 for DDO 167. Following Stasińska (1998), all the (semi)empirical methods have an associated minimal uncertainty of $0.1 \mathrm{dex}$. This uncertainty should be added to those derived from the line intensity ratios. In this case, the values are comparable to or larger than the uncertainties of the lowest quality data obtained with the sensitive-temperature method. Therefore, the method used by Pilyugin (2001) to derive the oxygen abundance is not the optimum one when high accuracy is needed as in the present study (see also Skillman et al. 2002).

\subsection{A linear trend $\log (O / H)-M_{b}$ as the result of sampling plus scattering}

The question that arises is whether the sampling of a family of nonlinear curves in the $Z-L$ plane may result in a linear log $(\mathrm{O} / \mathrm{H})-M_{\mathrm{b}}$ trend. For simplicity and to make the results reproducible we will assume that the $Z-L$ relationship is given by a function identical to Eq. (7), but it is clear that the conclusions hold true in the general case. In Fig. 8 a realization of 20 objects letting $b, q$ and $M_{\mathrm{b}}$ to be randomly distributed between 1 and 3, 0.2 and 0.95 and between -20 and -10 , respectively, is shown. A linear correlation between $\log (\mathrm{O} / \mathrm{H})$ and $M_{\mathrm{b}}$ is apparent. This is in part due to an artifact of the $\log -\log$ plot together with a poor sampling. The observational data-points are also plotted in this figure. The similarities between both trends are remarkable; the reason is that when all the parameters are allowed to vary, a linear trend between $\log (\mathrm{O} / \mathrm{H})$ and $M_{\mathrm{b}}$ is expected, but with high scatter. Note that the mentioned scatter is not a consequence of uncertainties in their determinations, but inherent to the different histories of the galaxies. However, large uncertainties in the measurements of the metallicity of low-luminosity galaxies contribute indirectly to the scattered appearance of the diagram.

\section{Conclusions}

The fact that dwarf galaxies are small systems have led to think that heavy elements could be more easily ejected to the intergalactic medium than in more massive galaxies. Nowdays, there is still some debate on the efficiency of this ejection even for low-mass galaxies. It is expected that the efficiency of loss of heavy elements should decrease with increasing luminosity from dwarfs to spirals, resulting in a tight correlation between metallicity and luminosity. However, if only dI galaxies are considered, high scatter in the $Z-L$ plane is likely to occur, as many competitive effects may be at play. The crude closed box model is commonly used to interpret the observations even in recent studies (e.g. Lee et al. 2002; Skillman et al. 2002). However, from this model little information can be obtained, since the relevant physics is hidden in the effective yield.

In this work we take advantage of the metallicities and luminosities of a sample of dI and Magellanic irregular galaxies selected by a strict criterion (see Paper I). The following conclusions can be inferred from the study presented here:

1- For a given set of parameters, the relationship between $Z$ and $L$ is not linear but saturates exponentially according to various simple chemical evolutionary models. The observational data are fitted well for reasonable variations of the parameters. The extreme values of these parameters required to account for the very low metallicity galaxies agree with the small number of these galaxies found, despite the numerous surveys carried out.

2- A linear correlation between the metallicity and the luminosity for a sample of dwarf irregular, non-bursting, galaxies, could be feasible for galaxies with luminosities $\leq 0.5 \times$ $10^{8} L_{\odot}$. However, observations show that this is not the case as the correlation between these two parameters gets weaker at low luminosities (see also RM).

3- A linear relationship between $12+\log (\mathrm{O} / \mathrm{H})$ and $M_{\mathrm{b}}$ may be a consequence of a poor sampling in a $\log -\log$ plot.

4- Variations in the stellar mass-to-light ratio can contribute significantly to the scatter in the $Z-L$ diagram, and the action of a mechanism of regulation between accretion and outflows cannot be ruled out.

5- We point out that tight linear correlations between $L$ and $Z$ found in previous works cannot be the result of a selection effect, with only gas-dominated galaxies in their samples. The samples of galaxies used in SKH, RM and Paper I contain similar amounts of gas-dominated galaxies while the trends obtained are very different.

6- Pilyugin (2001) obtained a linear $Z-L$ correlation using a semi-empirical method for the metallicity determination. 
We have also studied variations in the metallicity using semiempirical methods for its determination. The stardard sensitivetemperature method and the (semi)empirical(s) agree well for a metallicity larger than $7.9 \times 10^{-5}$, but they differ by up to 0.5 dex at low metallicity values. This fact could conspire in favor of a non-realistic linear correlation.

It is important to stress the necessity of high quality data on low metallicity and luminosity galaxies such as DDO 167 , DDO 155 and DDO 168. They are among the very low metallicity galaxies but the associated uncertainties are large. It is necessary to obtain smaller uncertainties in order to know if they are placed on their proper position in the $Z-L$ plane. This could help us to understand the ejection of metal in low-mass galaxies. On the other hand, more and accurate values of metallicities are needed for high metallicity and high luminosity galaxies in order to extend the observational sample into this region.

We warn that the Eq. (43) of Ferrara \& Tolstoy (2000) contains a wrong extra-factor $1-Z$, and that this mistake has been propagated throughout that paper. It is difficult to say whether the numerical model was affected by this fact, but we feel that a quantitative interpretation of their results is still lacking.

Acknowledgements. A.M.H-G. had been financially supported by NOTSA and by UAO. J.-R. Roy and J. Sommer-Larsen are thanked for many fruitful discussions of a first version of this article. A critical referee report has improved the presentation and material of this paper. A.M.H-G. thanks IA-UNAM for their hospitality and L. Binette for support by the CONACyT grant 32139-E. F.J.S.S. acknowledges a postdoctoral grant from CONACyT.

\section{Appendix A: Solving the equations of the chemical evolution. Constant mass of gas in the galaxy}

As mentioned in Sect. 2, the starting points are the general equations of the chemical evolution. They are written in Ferrara \& Tolstoy (2000; hereafter FT) as

$\frac{\mathrm{d} m_{\mathrm{g}}}{\mathrm{d} t}=-\psi+E+A-W$

and

$\frac{\mathrm{d}\left[m_{\mathrm{g}} Z\right]}{\mathrm{d} t}=-Z[\psi+W]+E_{i}+Z^{\prime} A$

where $\psi, A, W, E$ and $Z^{\prime}$ are also functions of $t$.

Substituting $E=R \psi$ and $E_{i}=\left[R Z+y_{z}(1-R)\right] \psi$, these equations can be written as follows,

$\frac{\mathrm{d} m_{\mathrm{g}}}{\mathrm{d} t}=(R-1) \psi+A-W$

and

$\frac{\mathrm{d}\left[m_{\mathrm{g}} Z\right]}{\mathrm{d} t}=-Z[\psi+W]+R Z \psi+y_{z}(1-R) \psi+Z^{\prime} A$.

The first assumption to be made is that the mass of gas within the galaxy does not suffer appreciable variations with time (see
Sect. 2). Thus, from Eq. (A.3), follows $A-W=(1-R) \psi$. Furthermore, $\mathrm{d}\left(Z m_{\mathrm{g}}\right) / \mathrm{d} t=m_{\mathrm{g}} \mathrm{d} Z / \mathrm{d} t$ and Eq. (A.4) will become

$m_{\mathrm{g}} \frac{\mathrm{d} Z}{\mathrm{~d} t}=y_{z}(1-R) \psi+\left(Z^{\prime}-Z\right) A$.

In addition, from mass conservation with $m_{\mathrm{g}}=$ const., $\mathrm{d} m_{\mathrm{t}} / \mathrm{d} t=A-W$ and, therefore

$\frac{\mathrm{d} v}{\mathrm{~d} t}=\frac{1}{m_{\mathrm{g}}} \frac{\mathrm{d} m_{\mathrm{t}}}{\mathrm{d} t}=\frac{A-W}{m_{\mathrm{g}}}$

which defines the implicit dependence $v(t)$. Since $A>W, v$ is a monotonically increasing function of $t$. Dividing these two equations, the left hand side reads

$\left(m_{\mathrm{g}} \frac{\mathrm{d} Z}{\mathrm{~d} t}\right)\left(m_{\mathrm{g}} \frac{\mathrm{d} v}{\mathrm{~d} t}\right)^{-1}=\frac{\mathrm{d} Z}{\mathrm{~d} v}$.

The right side takes the form

$\frac{(1-R) y_{z} \psi}{A-W}+\left(Z^{\prime}-Z\right) \frac{A}{A-W}=y_{z}+\left(Z^{\prime}-Z\right) \frac{A}{A-W}$

where we have used $A-W=(1-R) \psi$. The equation for $Z(v)$ reduces to

$\frac{\mathrm{d} Z}{\mathrm{~d} v}=y_{z}+\left(Z^{\prime}-Z\right) \frac{A}{A-W}=y_{z}+\left(Z^{\prime}-Z\right) b$.

In the most general situation $b(v)$ and the formal solution is

$$
\begin{aligned}
Z(v)= & \exp [-B(v)] \\
& \times\left(\int\left(y_{z}+Z^{\prime} b\right) \exp [B(v)] \mathrm{d} v+C_{0}\right)
\end{aligned}
$$

where $B(v)$ is the primitive function of $b(v)$, i.e. $B(v) \equiv$ $\int^{v} b(v) d v$ and $C_{0}$ the constant of integration.

In the simplest case when $b$ is a constant, i.e. independent of $v$, the solution is

$Z(v)=C_{0} \exp \left(-\frac{A}{A-W} v\right)+y_{z} \frac{(A-W)}{A}+Z^{\prime}$.

The integration constant is inmediately obtained assuming that at $v=0$, the gas of the galaxy has a primordial composition. Thus, $C_{0}=\left(-y_{z}(A-W) / A\right)-Z^{\prime}$ and the final solution is

$Z(v)=\left(Z^{\prime}+y_{z} \frac{A-W}{A}\right)\left(1-\exp \left(-\frac{A}{A-W} v\right)\right)$

as written in Eq. (4) of Sect. 2.

\section{Appendix B: Solving the equations of the chemical evolution. Constant wind and no accretion}

If the accretion of mass is neglected as assumed by FT, Eqs. (A.1) and (A.2) become

$\frac{\mathrm{d} m_{\mathrm{g}}}{\mathrm{d} t}=(R-1) \psi-W$

and

$m_{\mathrm{g}} \frac{\mathrm{d} Z}{\mathrm{~d} t}=y_{z}(1-R) \psi$

We note that in FT this equation contains a wrong extra-factor $1-Z$, even though these authors are aware of the previous work 
by Maeder (1992) who already noticed that this mistake had been propagated in the literature. As a consequence, Eq. (44) of FT should say, in our notation,

$Z(t)=Z(0)+\frac{(1-R) y_{z}}{\tau t_{\mathrm{ff}}} t$

where $t_{\mathrm{ff}}$ is the galactic free-fall time and $\tau$ is an empirical correction factor. In their Eq. (45) there is also an extra-factor $1-Z$ in the right hand side. So that, assuming $W=0$, their Eq. (46) should read

$Z\left(m_{\mathrm{g}}\right)=Z(0)-y_{z} \ln \left(\frac{m_{\mathrm{g}}}{m_{\mathrm{g}}(0)}\right)$

where $m_{\mathrm{g}}(0)$ is the initial mass of the galaxy. This equation represents the classical closed-box model. Finally, for the case of constant wind,

$Z\left(m_{\mathrm{g}}\right)=Z(0)-y_{z} \ln \left(\frac{K m_{\mathrm{g}}-1}{K m_{\mathrm{g}}(0)-1}\right)$

where $K \equiv(1-R) / \tau t_{\mathrm{ff}} W$, and replace their Eq. (47).

Ferrara $\&$ Tolstoy (2000) end up with a linear correlation between the metallicity and the final mass of gas (see their Fig. 4) for a certain dark-to-visible mass ratio $\phi$. In the light of the above equations, the results of FT are difficult to interpret. We have compared the expected metallicity for two cases, both with $\phi=30$, but different final mass of gas $10^{8} M_{\odot}$ (case A) and $10^{9} M_{\odot}$ (case B), respectively. The values for the time-averaged mass outflow rate and star formation rate are $\sim 4 \times 10^{-5} M_{\odot} \mathrm{yr}^{-1}$ and $\sim 10^{-2} M_{\odot} \mathrm{yr}^{-1}$, respectively, for case A, and $\sim 10^{-4} M_{\odot} \mathrm{yr}^{-1}$ and $\sim 6 \times 10^{-2} M_{\odot} \mathrm{yr}^{-1}$, for case B. In both cases, $K m_{\mathrm{g}}$ is much larger than $1\left(K m_{\mathrm{g}} \sim 10^{2}\right)$ and Eq. (B.5) simplifies to:

$Z\left(m_{\mathrm{g}}\right)=Z(0)-y_{z} \ln \left(\frac{m_{\mathrm{g}}}{m_{\mathrm{g}}(0)}\right)$.

If $\tau_{\text {life }}$ is the present time of a galaxy, typically $12 \mathrm{Gyr}$, the initial mass of the galaxy is estimated as $m_{\mathrm{g}}(0)=m_{\mathrm{g}}\left(\tau_{\text {life }}\right)+$ $\tau_{\text {life }}[W+\psi /(1-R)]$. With the guide numbers given above, we get $Z\left(\tau_{\text {life }}\right)=Z(0)+y_{z} \ln [1+1.2 /(1-R)]$ for case $\mathrm{A}$, and $Z\left(\tau_{\text {life }}\right)=Z(0)+y_{z} \ln [1+0.7 /(1-R)]$ for case $\mathrm{B}$. The expected trend that the metallicity should be higher for smaller $m_{\mathrm{g}}$ is at sharp contrast with the results of Fig. 4 of FT. In other words, in order to have high metallicities for large $m_{\mathrm{g}}$, one would expect the higest star formation rates for the highest $m_{\mathrm{g}}$, provided $K m_{\mathrm{g}} \gg 1$. Therefore, it seems that either there is something subtle in their model or the description of the model is not completely transparent.

\section{References}

Aaronson, M., \& Mould, J. 1985, ApJ, 290, 191

Aparicio, A., \& Tikhonov, N. 2000, AJ, 119, 2183

Campos-Aguilar, A., Moles, M., \& Masegosa, J. 1993, AJ, 106, 1784

Chandar, R., Bianchi, L., \& Ford, H. C. 2000, AJ, 120, 3088

Chiosi, C. 1986, 16th Advance Course of the SAAS-FEE: Nucleosynthesis and Chemical Evolution, ed. B. Hauck, A. Maeder, \& G. Meynet, 199
Cole, S., Aragón-Salamanca, A., Frenk, C. S., Navarro, J. F., \& Zepf, S. E. 1994, MNRAS, 271, 781

Devost, D., Roy, J.-R., \& Drissen, L. 1997, ApJ, 482, 765

Dohm-Palmer, R. C., Skillman, E. D., Gallagher, J., et al. 1998, AJ, 116,1227

Efstathiou, G. 2000, MNRAS, 317, 697

Ferrara, A., \& Tolstoy, E. 2000, MNRAS, 313, 291 (FT)

Gallagher, J. S. III, \& Hunter, D. 1984, ARA\&A, 22, 37

Gallagher, J. S. III, \& Wyse, R. F. G. 1994, PASP, 106, 1225

Greggio, L., Marconi, G., Tosi, M., \& Focardi, P. 1993, AJ, 105, 894

González-Delgado, R. M., Pérez, E., Tenorio-Tagle, G., et al. 1994, ApJ, 437, 239

González-Riestra, R., Rego, M., \& Zamorano, J. 1988, A\&A, 202, 27

Hidalgo-Gámez, A. M. 1999, Ph.D. Thesis, Uppsala University

Hidalgo-Gámez, A. M., \& Olofsson, K. 1998, A\&A, 334, 45 (Paper I)

Hidalgo-Gámez, A. M., Olofsson, K., \& Masegosa, J. 2001a, A\&A, 367,388

Hidalgo-Gámez, A. M., Masegosa, J., \& Olofsson, K. 2001b, A\&A, 369,797

Hidalgo-Gámez, A. M., \& Olofsson, K. 2002, A\&A, 389, 836

Hidalgo-Gámez, A. M., \& Sánchez-Salcedo, F. J. 2002, Galaxies evolution: Theory and Observations, ed. V. Avila, C. Firmani, C. Frenk, \& C. Allen (Rev. Mex. AASC)

Huchtmeier, W. K., \& Richter, O. G. 1988, A\&A, 210, 1

Kennicutt, R. C. Jr. 1983, ApJ, 272, 54

Kennicutt. R. C. Jr. 1989, ApJ, 344, 685

Kennicutt R. C. Jr. 1998, ApJ, 498, 541

Kinman, T. D., \& Davidson, K. 1981, ApJ, 243, 127

Kobulnicky, H. A., \& Skillman, E. D. 1996, ApJ, 471, 211

Lee, H., McCall, M., Kingsburgh, R. L., et al. [astro-ph/0210098]

Lequeux, J., Rayo, F. J., Serrano, A., et al. 1979, A\&A, 80, 155

Mac Low, M.-M., \& Ferrara, A. 1999, ApJ, 513, 192

Maeder, A. 1992, A\&A, 263, 129

Maeder, A. 1993, A\&A, 268, 833

Martin, C. L., \& Kennicutt, R. C. Jr. 2001, ApJ, 555, 301

Masegosa, J., Moles, M., \& del Olmo, A. 1991, A\&A, 249, 505

Masegosa, J., Moles, M., \& Campos-Aguilar, A. 1994, ApJ, 420, 576

Mateo, M. L. 1998, ARA\&A, 36, 435

Matteucci, F., \& Chiosi, C. 1983, A\&A, 123, 121

McCall, M. L., Rybski, P. M., \& Shields, G. A. 1985, ApJS, 57, 1

McGaugh, S. S. 1994, ApJ, 426, 135

Moles, M., Aparicio, A., \& Masegosa, J. 1990, A\&A, 228, 310

Olofsson, K. 1997, A\&A, 321, 29

Osterbrock, D. E. 1989, Astrophysics of Gaseous Nebulae and Active Galactic Nuclei (University Science Books, Mill Valley, CA)

Pagel, B. E. J., Edmunds, M. G., Blackwell, D. E., et al. 1979, MNRAS, 189, 95

Peimbert, M., \& Serrano, A. 1982, MNRAS, 198, 563

Peña, M., \& Ayala, S. 1993, Rev. Mex. Astron. Astrofis., 27, 171

Pilyugin, L. S., \& Ferrini, F. 2000a, A\&A, 354, 874

Pilyugin, L. S., \& Ferrini, F. 2000b, A\&A, 358, 72

Pilyugin, L. S. 2001, A\&A, 374, 412

Puche, D., Carignan, C., \& Wainscoat, R. J. 1991, AJ, 101, 447

Recchi, S., Matteucci, F., \& D'Ercole, A. 2001, MNRAS, 322, 800

Richer, M. G., \& McCall, M. L. 1995, ApJ, 445, 642 (RM)

Salzer, J. 1998, XXXIII Rencontre de Moriond, Dwarf galaxies and Cosmology, ed. T. Thuan, Ch. Balkowski, V. Cayatte, \& J. T. Thanh Van (Éditions Frontières), 21

Silich, S., \& Tenorio-Tagle, G. 2001, ApJ, 552, 91

Skillman, E. D. 1989, ApJ, 347, 883

Skillman, E. D. 1999, Stellar Astrophysics for the Local Group, ed. A. Aparicio, A. Herrero, \& F. Sánchez (Cambridge Contemporary Astrophysics), 463 
Skillman, E. D., Kennicutt, R. C. Jr., \& Hodge, P. 1989, ApJ, 347, 875 (SKH)

Skillman, E. D., \& Kennicutt, R. C. Jr. 1993, ApJ, 411, 655

Skillman, E. D., Cotè, S., \& Miller, B. 2002 [astro-ph/0211172]

Somerville, R. S., \& Primack, J. R. 1999, MNRAS, 310, 1087

Stasińska, G. 1998, XXXIII Rencontre de Moriond, Dwarf Galaxies and Cosmology, ed. T. Thuan, Ch. Balkowski, V. Cayatte, \& J. T. Thanh Van (Éditions Frontières), 259

Strickland, D. K., \& Stevens, I. R. 2000, MNRAS, 314, 511
Tinsley, B. M. 1980, Fund. Cosm. Phys., 5, 287

van Zee, L., Haynes, M. P., \& Salzer, J. J. 1997, AJ, 114, 2497

Vigroux, L., Stasińska, G., \& Comte, G. 1987, A\&A, 172, 15

Vila-Costas, M. B., \& Edmunds, M. G. 1992, MNRAS, 259, 121

Walsh, J. R., \& Roy, J.-R. 1989, ApJ, 341, 722

Walsh, J. R., \& Roy, J.-R. 1993, MNRAS, 262, 27

Webster, B. L., Longmore, A. J., Hawarden, T. G., \& Mebold, U. 1983, MNRAS, 205, 643

Wyder, T. K. 2001, AJ, 122, 2490 